CER: - TIS gS-P RP. FF

EUROPEAN LABORATORY FOR PARTICLE PHYSICS

$8+5544$

CERN LIBRARIES, GENEVA

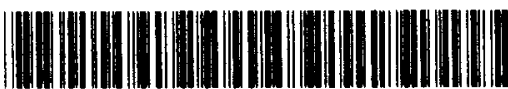

CERN/TIS-RP/95-18/PP

CERN-TIS-95-18

\title{
Investigation of the PDM-303 Neutron Pocket Dosemeter in Various Neutron Fields
}

\author{
A. Aroua ${ }^{1}$ and M. Höfert ${ }^{2}$ \\ ${ }^{1}$ IAR, Institute for Applied Radiophysics, CH-1015 Lausanne, Switzerland. \\ ${ }^{2}$ CERN, European Laboratory for Particle Physics, CH-1211 Genève 23, Switzerland.
}

\begin{abstract}
Following a calibration by Physikalisch-Technische Bundesanstalt (PTB) of the Aloka PDM303 neutron pocket dosemeter in an energy range from thermal to neutrons of $19 \mathrm{MeV}$ the response of the instrument has been further investigated in various neutron fields. Measurements were performed at the Paul Scherrer Institut (PSI) in nearly monoenergetic neutron beams of 35 and $55 \mathrm{MeV}$. Furthermore, the response has been evaluated experimentally in well characterized neutron fields at the Cadarache calibration facility and at the CERN high-energy calibration facility. The experimental results are compared with calculated values established by convoluting the known response function of the detector with the spectra of the neutron fields. The results indicate that the dosemeter is a promising active instrument for personnel dosimetry, particularly in high-energy fields, but that efforts should still be made to improve its dose equivalent response.
\end{abstract}

To be submitted to Nuclear Instruments and Methods (NIM A)

October 1995 


\section{Introduction}

The "MYDOSE-mini" PDM-303 model pocket electronic dosemeter [1], produced by the Japanese company Aloka Co. Ltd, allows the measurement of the integrated neutron dose equivalent and indicates it on a digital display. It consists of a single sensor Si-photodiode, makes use of the nuclear reaction ${ }^{6} \mathrm{Li}+\mathrm{n} \rightarrow \alpha+{ }^{3} \mathrm{~T}$ and is sensitive to neutrons only [2]. The PDM-303 is designed to measure neutrons from thermal energies up to about $15 \mathrm{MeV}$ [1]. Fast neutrons are detected by means of the albedo method. The dosemeter has a convenient size (145 mm $\times 30 \mathrm{~mm} \times 12 \mathrm{~mm}$ ) and weighs only about $70 \mathrm{~g}$.

During the past years the PDM-303 dosemeter has been investigated by several laboratories [3-5] such that its dose equivalent response function is now accurately known in the neutron energy range from thermal to $19 \mathrm{MeV}$.

This paper presents the results of the investigation carried out on two prototypes of the PDM-303 (Nos. X0084 and X0097) that Aloka has offered to CERN for test purposes. This investigation covers the calibration of the PDM-303 with monoenergetic neutrons of 35 and $55 \mathrm{MeV}$, and measurements performed in three different, well characterized, neutron fields.

\section{Measurement conditions}

\subsection{The PSI calibration beams}

The dosemeter was calibrated at the Paul Scherrer Institut (PSI), Villigen, with quasi monoenergetic neutron beams of 35 and $55 \mathrm{MeV}$, produced at the injecting cyclotron, in bombarding a $2 \mathrm{~mm}$ thick ${ }^{9} \mathrm{Be}$ target with protons of 40 and $65 \mathrm{MeV}$ respectively. The diameter of the neutron beams was $40 \mathrm{~mm}$. A detailed description of the production of the neutron beams and their monitoring is given by Schuhmacher and Alberts [6].

\subsection{The Cadarache realistic neutron calibration field}

The Cadarache realistic neutron calibration field simulates a situation that is typically encountered in operational radiation protection at nuclear reactors. The field is produced 
using a $14 \mathrm{MeV}$ generator, a uranium converter and different filters [7]. Its neutron spectrum is presented in figure $1[8]$.

\subsection{The CERN high-energy calibration fields}

The CERN high-energy reference fields are produced in the North experimental hall with a secondary hadron beam from the Super Proton Synchrotron (SPS) [9]. This beam of 205 $\mathrm{GeV} / \mathrm{c}$ hadrons (protons and pions) interacts with a $50 \mathrm{~cm}$ long, $7 \mathrm{~cm}$ diameter copper target. The secondary radiation emitted at large angles up to $90^{\circ}$ from this target passes through shields of either $80 \mathrm{~cm}$ of concrete or $40 \mathrm{~cm}$ of iron. The radiation arrives in bursts of $2.5 \mathrm{~s}$ duration with a repetition time of $14.4 \mathrm{~s}$. Two measurements were carried out, one on the concrete and the second on the iron shielding. The neutron spectra of these fields are shown in figure $1[10]$.

\subsection{Irradiation geometry}

At the PSI the PDM-303 was exposed on a plexiglas phantom with dimensions : $10 \times 10 \times 5$ $\mathrm{cm}^{3}$, at Cadarache on a plexiglas phantom with standard dimensions : $30 \times 30 \times 15 \mathrm{~cm}^{3}$, and at CERN on a polyethylene pseudo-sphere of $25 \mathrm{~cm}$ diameter.

\section{Results and discussion}

A detailed evaluation of the results of the calibration irradiations at PSI is given elsewhere [4]. Table 1 summarizes the results for the PDM-303. The reference value is based on the asssumption of monoenergetic neutrons in the beams, their fluence determination, and on fluence to dose equivalent conversion factors according to the work of Schuhmacher and Alberts [6]. Both dosemeters apparently overrespond to high-energy neutrons while the agreement between the reading of the two detectors is excellent at $35 \mathrm{MeV}$, and within less than $15 \%$ at $55 \mathrm{MeV}$.

Figure 2 shows the response curve for the PDM-303 established by PTB to which the results given in table 1 have been added. The whole set of data was fitted by a spline function to 
establish the continuous curve shown in figure 1 and which is going to be refered to as the dose equivalent response function of the dosemeter.

The results of the measurements at Cadarache are summarized in table 2 . The agreement between the two detectors is good, within less than $5 \%$. However, they overestimate the dose equivalent in the neutron field by more than a factor of 7 . Such a behaviour is expected when looking at the response curve in figure 2 . When convoluting the response function of the dosemeter given in figure 2 with the measured spectrum presented in figure 1 this overestimation should be even a factor of 14 (last column of table 2). This factor of 2 between the calculated and measured relative response appears to be nearly insignificant compared with the overall overestimation of the PDM-303 in typical neutron fields around reactors.

The results of the measurements at the CERN high-energy reference field facility are summarized in table 3 . The agreement between the two detectors, except in one case, is rather good. Here again the last column of table 3 gives the value of the dose equivalent response evaluated by convoluting the response function of the dosemeter given in figure 2 with the measured spectra (on concrete and iron shielding) presented in figure 1 . The agreement between measured and calculated relative response of the dosemeter is reasonable for the concrete shielding. For the iron shielding however, the two results are significantly different and, considering the neutron spectrum involved, incomprehensible. In the case of the iron shielding the total dose rates were about an order of magnitude higher than on the concrete shielding with an even higher neutron fluence in the intermediate energy range. Therefore, and in view of the pulsed nature of the radiation which furthermore leads to an increase in the instantaneous dose rate, a saturation effect of the detector cannot be excluded. In fact the manufacturer indicates a measurement range between 0.01 and 99.99 $\mathrm{mSv}$ (for a calibration with 4.5 MeV Am-Be neutrons, on a phantom). From figure 2 one sees that the relative response of the dosemeter at this energy is close to unity. However, for intermediate energies the relative response is up to two orders of magnitude higher. In this energy range the dosemeter is therefore likely to show problems of linearity not at $100 \mathrm{mSv}$ but already around 1 to $\ldots \mathrm{nSv}$ which would also explain the underestimation by a factor of 
two in the Cadarache field. At CERN the integrated dose equivalent for all measurements was between 4 and $6 \mathrm{mSv}$, and at Cadarache it was even higher (around $16 \mathrm{mSv}$ ). Further measurements in the intermediate energy range are necessary to clear up the behaviour of the dosemeter at high doses and dose rates.

\section{Conclusion}

The investigation described in this report shows that the PDM-303 neutron pocket dosemeter can be used in high-energy radiation stray fields around an accelerator if a certain overestimation is accepted. The overestimation noticed in high-energy radiation fields confirms observations when the instrument was carried on aircrafts during continental flights, where higher dose equivalents were obtained than could be reasonably expected [11]. A sensitivity to high-energy charged particles would explain the observed overestimation in high-energy stray fields.

While the response in high-energy fields is comprehensible and acceptable, the behaviour in neutron fields of lower energy is not well understood. In fact, considering the measured response function of the instrument a considerable overestimation is expected. This is not confirmed in the case of two practical neutron fields.

This active dosemeter must be considered useful for individual dosimetry, due to its small size and weight, if further efforts are made to improve its dose equivalent response.

\section{References}

[1] Electronic Pocket Dosimeter (EPD $\left.{ }^{\mathrm{TM}}\right)$, MYDOSE mini ${ }^{\mathrm{TM}}$, Model PDM-303, Instruction Manual, Aloka Co. Ltd., Mitaka-Shi, Tokyo.

[2] Oshima, T., Private Communaication (1993).

[3] Raffnsøe, C. R., Test of the Neutron Pocket Dosemeter PDM-303, Technical Memorandum CERN/TIS-RP/TM/92-20/Rev., European Laboratory for Particle Physics (CERN), Genève (1992). 
[4] Aroua, A., Quelques résultats de calibrations des dosimètres de poche Aloka, Technical Memorandum CERN/TIS-RP/TM/93-31, European Laboratory for Particle Physics (CERN), Genève (1993).

[5] Alberts, W.G., Dietz, E., Guldbakke, S. and Kluge, H., Response of an Electronic Personal Neutron Dosemeter, Radiat. Prot. Dosim. $51 / 3$ (1994) 207-210.

[6] Schuhmacher, H. and Alberts, W.G., Reference Neutron Fields with Energies up to 70 $\mathrm{MeV}$ for the Calibration of Radiation Protection Instruments, Radiat. Prot. Dosim. 42/4 (1992) 287-290.

[7] Chartier, J.-L., Posny, F. and Buxerolle, M., Experimental Assembly for the Simulation of Realistic Neutron Spectra, Radiat. Prot. Dosim. 44/1 (1992) 125-130.

[8] Chartier, J.-L., Private Communication (1995).

[9] Aroua, A., Höfert, M., Sannikov, A. V. and Stevenson G. R., Reference High-Energy Radiation Fields at CERN, Presented at the 6th International Symposium on Radiation Physics, Rabat, Morocco, 18-22 July 1994, Divisional Report CERN/TIS-RP/9412/CF, European Laboratory for Particle Physics (CERN), Genève (1994).

[10] Rösler, S. and Stevenson, G. R., July 1993 CERN-CEC Experiments : Calculations of Hadron Energy Spectra from Track-Length Distributions using FLUKA, Internal Report CERN/TIS-RP/IR/93-47, European Laboratory for Particle Physics (CERN), Genève (1993).

[11] Höfert, M., Private Communication (1995). 
Table 1. Dose equivalent response of the PDM-303 dosemeter to 35 and $55 \mathrm{MeV}$ neutrons (Dose equivalent in $\mathrm{nSv} /$ monitor unit)

\begin{tabular}{lcccccc}
\hline & \multicolumn{3}{c}{$\mathrm{E}_{\mathrm{n}}=35 \mathrm{MeV}$} & \multicolumn{3}{c}{$\mathrm{E}_{\mathrm{n}}=55 \mathrm{MeV}$} \\
Detector & Reference & Measurement & Meas./Ref. & Reference & Measurement & Meas./Ref. \\
\hline X0084 & 53.8 & 91.4 & 1.70 & 70 & 142 & 2.03 \\
X0097 & 53.8 & 91.1 & 1.69 & 70 & 126 & 1.80 \\
Mean & 53.8 & 91.2 & 1.69 & 70 & 134 & 1.91 \\
\hline
\end{tabular}


Table 2. Dose equivalent response of the PDM-303 at the Cadarache neutron field (Dose equivalent in $\mathrm{mSv}$ )

\begin{tabular}{lccc}
\hline Dosemeter & Reference & Measurement & Meas./Ref. \\
\hline X0084 & 2.2 & 16.6 & 7.5 \\
X0097 & 2.2 & 15.9 & 7.2 \\
Mean & 2.2 & 16.2 & 7.4 \\
Convolution & & & 13.8 \\
\hline
\end{tabular}


Table 3. Dose equivalent response of the PDM-303 at the CERN neutron fields (Dose equivalent in $10^{-10} \mathrm{~Sv} /$ monitor unit)

\begin{tabular}{|c|c|c|c|c|c|c|c|}
\hline \multicolumn{3}{|c|}{ Measurement 1} & \multicolumn{3}{|c|}{ Measurement 2} & \multirow{2}{*}{$\begin{array}{c}\text { Overall } \\
\text { mean }\end{array}$} & \multirow{2}{*}{$\begin{array}{c}\text { Convo- } \\
\text { lution }\end{array}$} \\
\hline Ref. & Meas. & Meas./ref. & Ref. & Meas. & Meas./ref. & & \\
\hline
\end{tabular}

Concrete shielding

\begin{tabular}{lllllllll}
\hline X0084 & 2.7 & 10.2 & 3.8 & 2.6 & 15.1 & 5.8 & & \\
X0097 & 2.6 & 15.9 & 6.1 & 2.7 & 15.5 & 5.7 & & \\
Mean & & & 4.9 & & & 5.8 & 5.4 & 3.6 \\
\hline Iron shielding & & & & & & & \\
\hline X0084 & 14.4 & 23.8 & 1.7 & 14.4 & 15.4 & 1.1 & & \\
X0097 & 12.9 & 25.6 & 2.0 & 12.9 & 16.8 & 1.3 & & \\
Mean & & 1.8 & & & 1.2 & 1.5 & 5.7 \\
\hline
\end{tabular}




\title{
Investigation of the PDM-303 Neutron Pocket Dosemeter in Various Neutron Fields
}

\author{
A. Aroua and M. Höfert
}

\section{FIGURE CAPTIONS}

Figure 1. Neutron fluence spectra of the fields used for the investigation of the PDM-303 Electronic Pocket Dosemeter.

Figure 2. Dose equivalent response of the PDM-303 Electronic Pocket Dosemeter. The empty symbols are the PTB calibration points [5]. The full symbols correspond to the PSI calibration points [4]. 

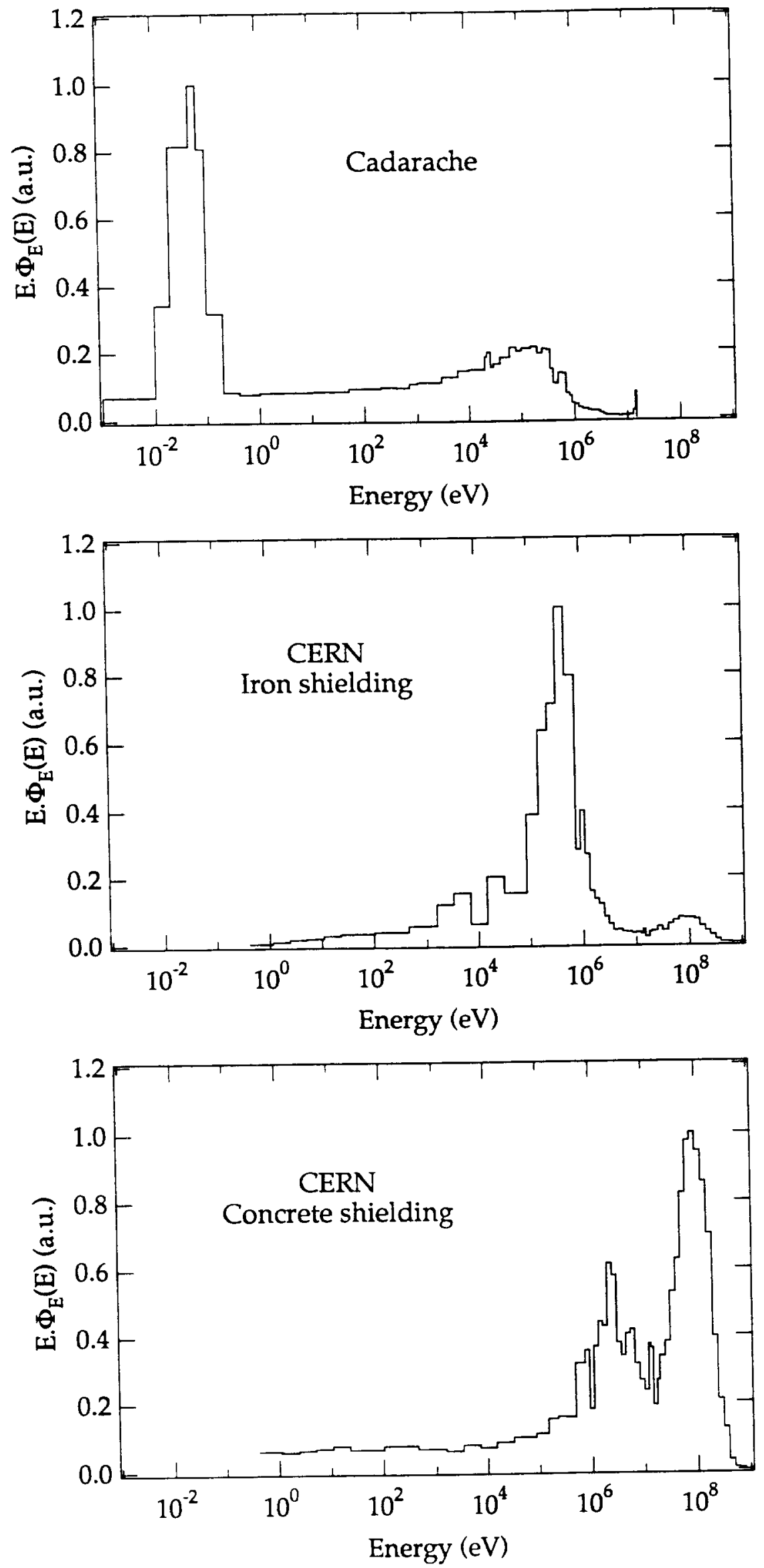

Figure 1. 


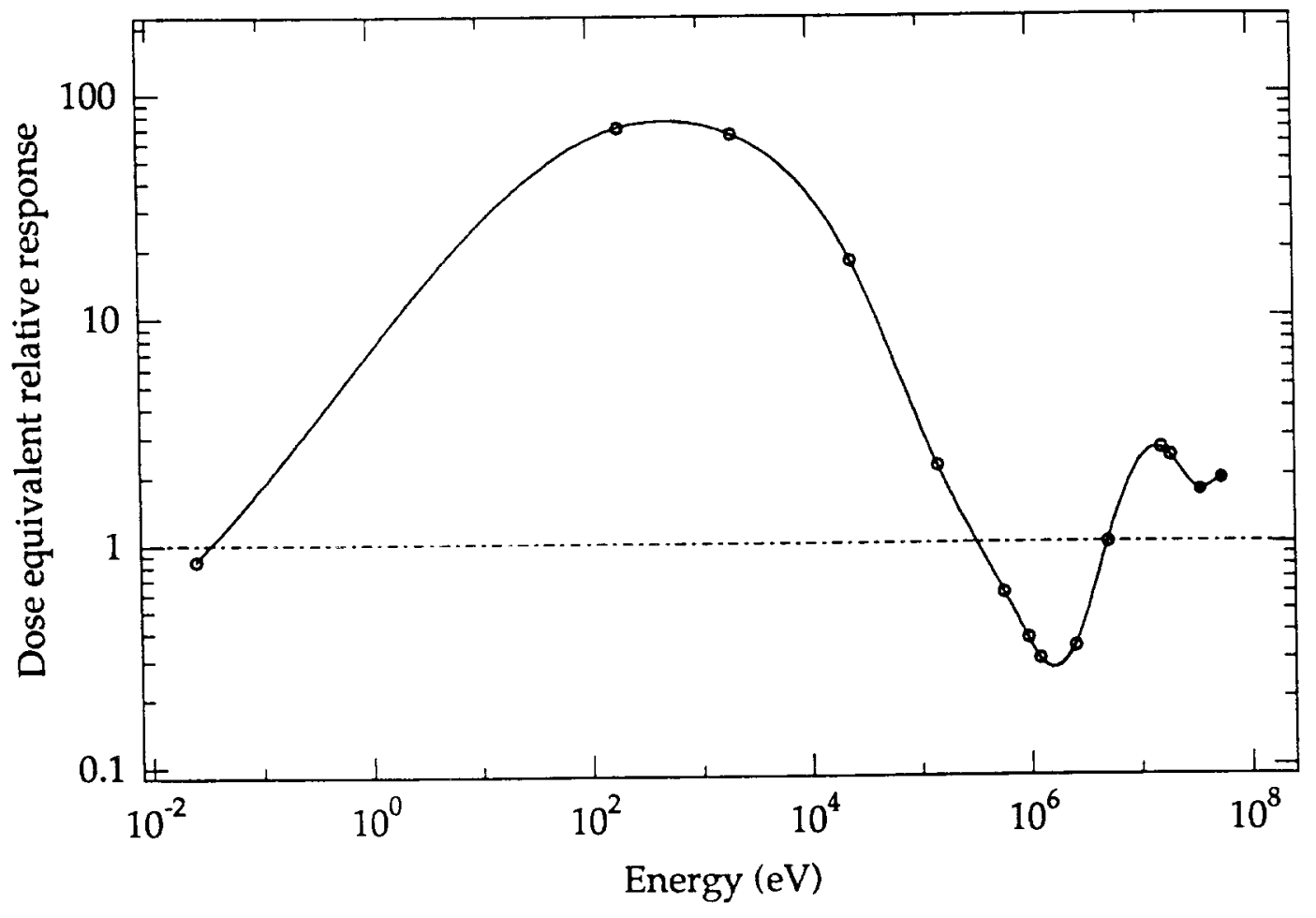

Finure 2 